\title{
A heuristic method to identify runs of homozygosity associated with reduced performance in livestock
}

\author{
J. T. Howard, $* 1$ F. Tiezzi, * Y. Huang, $\dagger$ K. A. Gray, $\uparrow$ and C. Maltecea* $*$ \\ *Department of Animal Science, North Carolina State University, Raleigh 27695-7627; †Smithfield Premium \\ Genetics, Rose Hill, NC 28458; and †Genetics Program, North Carolina State University, Raleigh 27695-7627
}

\begin{abstract}
Although, for the most part, genomewide metrics are currently used in managing livestock inbreeding, genomic data offer, in principle, the ability to identify functional inbreeding. Here, we present a heuristic method to identify haplotypes contained within a run of homozygosity $(\mathrm{ROH})$ associated with reduced performance. Results are presented for simulated and swine data. The algorithm comprises 3 steps. Step 1 scans the genome based on marker windows of decreasing size and identifies $\mathrm{ROH}$ genotypes associated with an unfavorable phenotype. Within this stage, multiple aggregation steps reduce the haplotype to the smallest possible length. In step 2, the resulting regions are formally tested for significance with the use of a linear mixed model. Lastly, step 3 removes nested windows. The effect of the unfavorable haplotypes identified and their associated haplotype probabilities for a progeny of a given mating pair or an individual can be used to generate an inbreeding load matrix (ILM). Diagonals of ILM characterize the functional individual inbreeding load (IIL). We estimated the accuracy of predicting the phenotype based on IIL. We further compared the significance of the regression coefficient for IIL on phenotypes with genome-wide
\end{abstract}

inbreeding metrics. We tested the algorithm using simulated scenarios (12 scenarios), combining different levels of linkage disequilibrium (LD) and number of loci impacting a quantitative trait. Additionally, we investigated 9 traits from 2 maternal purebred swine lines. In simulated data, as the LD in the population increased, the algorithm identified a greater proportion of the true unfavorable $\mathrm{ROH}$ effects. For example, the proportion of highly unfavorable true $\mathrm{ROH}$ effects identified rose from 32 to $41 \%$ for the low- to the high-LD scenario. In both simulated and real data, the haplotypes identified were contained within a much larger ROH (9.12-12.1 Mb). The IIL prediction accuracy was greater than 0 across all scenarios for simulated data (mean of 0.49 [95\% confidence interval $0.47-0.52]$ for the high-LD scenario) and for nearly all swine traits (mean of 0.17 [SD 0.10]). On average, across simulated and swine data sets, the IIL regression coefficient was more closely related to progeny performance than any genome-wide inbreeding metric. A heuristic method was developed that identified $\mathrm{ROH}$ genotypes with reduced performance and characterized the combined effects of ROH genotypes within and across individuals.

Key words: inbreeding, runs of homozygosity, swine
J. Anim. Sci. 2017.95:4318-4332 doi:10.2527/jas2017.1664

\section{INTRODUCTION}

The implementation of routine genotyping within livestock breeding populations has become a common practice and is used as a tool to make more effective selection decisions in swine breeding companies (Knol et al., 2016). Previous research has highlighted the

\footnotetext{
${ }^{1}$ Corresponding author: jthoward@ncsu.edu

Received April 25, 2017.

Accepted August 15, 2017.
}

advantages of genomic relationships compared with pedigree-based information to obtain more precise estimates of the genetic merit and homozygosity of an individual (Lopes et al., 2013; Knol et al., 2016). Also, genomic information allows genome-wide inbreeding estimates to be supplemented by characterizing the impact of homozygosity for specific genomic regions. Elevated levels of homozygosity result in a reduction in phenotypic performance, referred to as inbreeding depression (Falconer and Mackay, 1996). The identification of region-specific stretches allows breeders to more 
effectively manage inbreeding because the impact of homozygosity for a trait can vary across the genome. The estimation of dominance effects to identify unfavorable regions of the genome has been used in the past (Lopes et al., 2016; Xiang et al., 2016) but lacks power for lowfrequency mutations and does not consider that whole segments of the genome are passed from parent to offspring. To overcome these limitations, regions of the genome in a continuous run of homozygosity (ROH) have been proposed to investigate homozygous segments that arose due to past inbreeding (Howard et al., 2015; Saura et al., 2015). Previous research has investigated the phenotypic effect of a region being in a ROH (Pryce et al., 2014; Howard et al., 2015). The previous methods did not directly identify the unique $\mathrm{ROH}$ genotype that gave rise to the reduced phenotypic performance. Therefore, the objective of the current study was to develop a heuristic algorithm that identifies unfavorable haplotypes contained within $\mathrm{ROH}$ across the genome. The method was tested on simulated as well as real data.

\section{MATERIALS AND METHODS}

No animal care approval was required for this work because all genotypes and records came from data that were available from previous studies. The manuscript will be split in 2 sections. In the first section, an overview of the algorithm along with methods to summarize the number of unfavorable haplotypes shared within and across individuals is described. In the second section, simulated and swine data sets are used to summarize 3 major results: 1) how effective the algorithm is at identifying unfavorable haplotypes, 2) the length of $\mathrm{ROH}$ the unfavorable haplotype tags, and 3) the relationship of the aggregate effect of unfavorable haplotypes carried by an individual with its phenotype and genetic value.

\section{Description of the Algorithm}

A pictorial overview of the algorithm is displayed in Fig. 1. The method follows 3 steps. The first step scans the genome to identify $\mathrm{ROH}$ genotypes that result in an unfavorable change in the phenotype of interest. The genotypes used in the algorithm are coded as 0 for the homozygote, 1 for the heterozygote, and 2 for the alternative homozygote. Step 1 begins at the first SNP of a chromosome by constructing a window of a predetermined number of SNP (default $=60$ ). Within a window, the mean phenotype is tabulated for each unique $\mathrm{ROH}$ genotype and any genotype that is not in a $\mathrm{ROH}$ is aggregated into a category referred to as non- $\mathrm{ROH}$. A ROH genotype is not allowed to have any heterozygous genotypes. Furthermore, any ROH genotype below a user-defined frequency (default $=0.0075)$ is removed from the $\mathrm{ROH}$ genotype list and placed in the non- $\mathrm{ROH}$ category. If the phenotypic mean for a $\mathrm{ROH}$ genotype is below/above a user-defined value (discussed below), the window is stored. Next, the window is shifted forward by $1 \mathrm{SNP}$ and the previous process is repeated. Once the entire chromosome has been scanned, windows containing the same set of animals and representing the same ROH genotype except for the first and last SNP are aggregated (Fig. 1, step 1b). Because recombination does not occur within a given region for the individuals of the same $\mathrm{ROH}$ genotype, $\mathrm{ROH}$ genotypes that are combined in this step contain the same amount of information. Following the aggregation of nested windows, the window length is reduced by " $n$ " SNP (default = 5) and the previous steps are repeated for the new window size. The window size is reduced by " $n$ " until a minimum window size is reached (default $=20$ ). Once the minimum window size is reached, the process of scanning for unfavorable ROH genotypes is complete for a chromosome. For windows that contain the same set of animals and are nested within each other (Fig. 1, step 1d), the shortest window is kept for further analysis. The aggregation steps (Fig. 1, step $1 \mathrm{~b}$ and 1d) trap the $\mathrm{ROH}$ genotypes across individuals that have the same core ROH genotype to the smallest possible length. No information is lost within the aggregation steps because each $\mathrm{ROH}$ genotype belongs to the same set of individuals, yet the step significantly reduces the number of windows tested in following steps. The core ROH genotype is now expected to serve as a tag for the full $\mathrm{ROH}$ segment observed in an individual, which may differ across subjects due to recombination occurring at different locations across subjects. As a result, the length of the tag $\mathrm{ROH}$ genotype is expected to be short compared with the observed $\mathrm{ROH}$ genotype across individuals that have the same unique tag $\mathrm{ROH}$ genotype.

Any window remaining after step 1 is subsequently tested for significance using a standard mixed model that accounts for the environment and additive genetic and permanent environment effect of an individual plus any number of fixed effects. A description of the full model for each window is outlined below:

$$
y=\mathbf{X b}+\mathbf{Z a}+\mathbf{W p e}+\mathbf{e},
$$

in which $y$ is the trait of interest, $\mathbf{b}$ is a vector of fixed effects, $\mathbf{a}$ is a vector of random additive genetic effects, pe is a vector of random permanent environmental effects, $\mathbf{e}$ is a vector of random residuals, and $\mathbf{X}, \mathbf{Z}$, and $\mathbf{W}$ are incidence matrices relating $\mathbf{b}, \mathbf{a}$, and $\mathbf{p e}$, respectively, to $y$. The fixed effects can include any environmental classification or covariate effect along with the effect of $\mathrm{ROH}$ genotype (i.e., unique $\mathrm{ROH}$ genotype and non- $\mathrm{ROH}$ ) for a given window. The random 


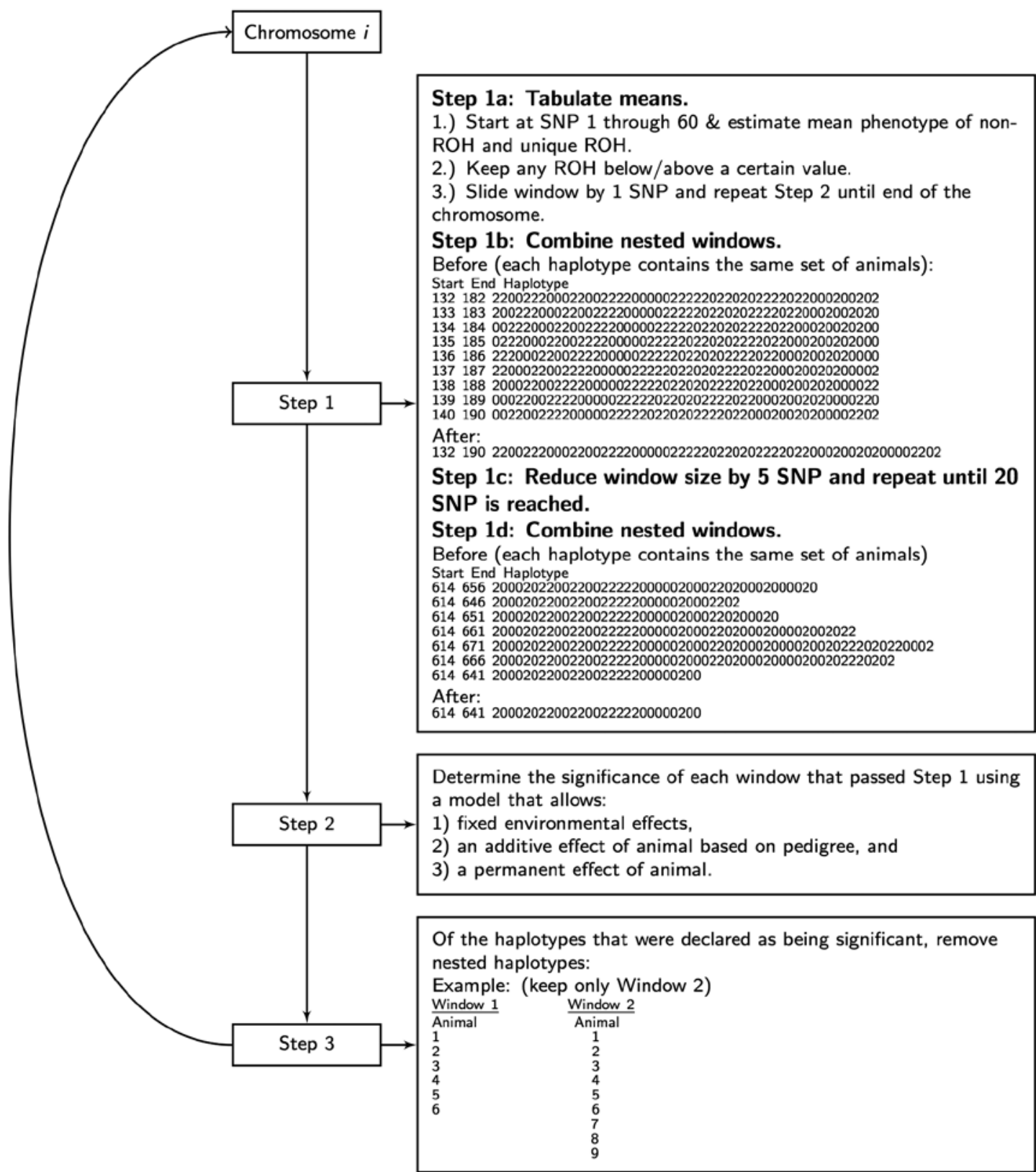

Figure 1. Overview of algorithm that identifies unfavorable haplotypes. $\mathrm{ROH}=$ run of homozygosity.

additive genetic effect is assumed $\sim N\left(0, \mathbf{A} \sigma_{\mathrm{a}}{ }^{2}\right.$, with $\mathbf{A}$ representing the additive relationship matrix derived from a pedigree (Henderson, 1976). The random permanent environmental and residual effects are assumed $\sim N\left(0, \mathbf{I} \sigma_{\mathrm{pe}}{ }^{2}\right)$ and $\sim N\left(0, \mathbf{I} \sigma_{\mathrm{e}}{ }^{2}\right)$, respectively, with $\mathbf{I}$ being an identity matrix. Variance components of the current implementation are assumed fixed across windows based on the null model of no ROH effect (i.e., no ROH genotype in the model). For each window, solutions are obtained using the Cholesky decomposition of the left-hand side. Given the solutions for each window, a contrast between each unique ROH genotype versus non-ROH and the associated $t$-statistic are obtained. The hypothesis test is 1-sided, and the direction of the test is dependent on the direction of the unfavorable phenotype. Under this parameterization, the genotypes not in a $\mathrm{ROH}$ are assumed normal compared with individuals that have the ROH genotype. This aligns with the partial dominance hypothesis, which is thought to account for the majority of inbreeding depression observed in populations (Simmons and Crow, 1977; Charlesworth and Charlesworth, 1987). Any contrast that passes the user-defined significance threshold is kept and moved onto the final window reduction step, which resolves nested windows (Fig. 1, step 3).

The algorithm presented was developed in $\mathrm{C}++11$. The source code and compiled executable files for Linux operating systems are available at https://github.com/ 
jeremyhoward. The primary option the user controls is the cutoff value for the mean phenotype for a given $\mathrm{ROH}$ genotype that is considered unfavorable in Fig. 1, stepla. The user can specify a cutoff value based on prior knowledge of what is considered an unfavorable phenotype or generate an empirical $t$-statistic distribution from the data to declare a cutoff value. The latter option is conducted by randomly specifying a chromosome, window length, and start position and estimating the significance value for $\mathrm{ROH}$ genotypes within the window. All 1 -sided $t$-statistics are stored. Across samples, the mean phenotype for $t$-statistics with a significance ranging from 0.05 to 0.10 is chosen as the cutoff value.

The haplotypes identified can be used in a variety of ways, but 2 are investigated in the current study. The first application is to apply the algorithm across economically important phenotypes and identify haplotypes having an unfavorable effect across multiple traits. Regions with consistent unfavorable effect across multiple traits should have a high probability of being sensitive to inbreeding and therefore result in a reduction in the overall fitness and vigor of an individual. The second application is to generate a matrix aiming at characterizing the decrease in the trait of interest across all unfavorable haplotypes, herein referred to as the inbreeding load matrix (ILM). Its calculation follows the method outlined by Cole (2015). To implement an ILM, the genotype phase needs to be known. This matrix then can be used in mating designs to minimize the probability of progeny containing the unfavorable haplotype or haplotypes for a single trait or across multiple traits. The diagonals of the matrix, referred to as individual inbreeding load (IIL), represent an individual's decrease in phenotypic performance due to inbreeding, whereas off-diagonals represent the predicted decrease in phenotypic performance in the trait of the progeny given the mating of the 2 (potential) parents. It should be noted that in this implementation, the algorithm does not simultaneously run all haplotypes across the genome. As a result, any observed $\mathrm{ROH}$ genotype for an individual might contain multiple significant unfavorable haplotypes. Therefore, multiple tag haplotypes identified by the algorithm could be counted as different in an individual when in fact the unfavorable haplotypes are tagging the same observed haplotype. Within the current study, when multiple haplotypes tagged the same observed $\mathrm{ROH}$ genotype, only the haplotype with the highest significance value and resulting in the largest number of haplotypes observed across individuals was retained. For the $i$ th row and $j$ th column of the ILM, the following formula was used to calculate elements of the ILM:

$$
\mathrm{ILM}_{i j}=\sum_{h=1}^{h=n} \mathrm{ROH}_{h} \times \hat{\beta}_{h},
$$

in which $n$ is the number of unfavorable haplotypes that remained after eliminating haplotypes that were not observed or removed to avoid double counting The $\mathrm{ROH}_{h}$ refers to the probability of generating a $\mathrm{ROH}$ for haplotype $h$ and $\beta_{h}$ is the effect of the ROH genotype estimated from step 2 of the algorithm. The probability values for the diagonals elements of the ILM include 0.25 (haplotype carrier) or 1.0 (haplotype in $\mathrm{ROH})$. The probability values for the off-diagonal elements include 0.25 (mating of haplotype carriers), 0.5 (mating of haplotype carrier and ROH genotype), or 1.0 (both parents have $\mathrm{ROH}$ genotype). The ILM values range from 0 (i.e., no unfavorable haplotypes) to any value in the unfavorable direction.

\section{Summary of Metrics Used to Test the Algorithm using Simulated and Swine Data}

Simulated data sets, where the true genetic signal is known, were used to determine how effective the algorithm was at identifying true negative $\mathrm{ROH}$ regions as well as to characterize the relationship between IIL and the true aggregate genotypic value of individuals. The length of ROH the unfavorable haplotype tagged was determined across both simulated and swine data sets to ensure that long stretches of $\mathrm{ROH}$ were represented. Long $\mathrm{ROH}$ stretches have a higher probability of being true identical by descent (IBD) segments as a result of recent inbreeding, compared with shorter ones. The relationship between IIL and the phenotype was summarized based on either 1) IIL accuracy of predicting the phenotype or 1) the significance of the regression coefficient when IIL was included as a fixed covariate in a mixed linear model. The latter relationship was generated under the premise that the management of inbreeding is traditionally done by minimizing parental coancestries using genome-wide inbreeding metrics. Therefore, the significance (i.e., $-\log P$-value) of the regression coefficient from traditionally used genome-wide inbreeding metrics was compared with the IIL value. Lastly, IIL was benchmarked across simulated and swine data sets with estimates of the genetic value based on a whole-genome regression model. This was conducted to generate a reference comparison on the prediction accuracy for a given trait based on traditionally used genome-wide modeling techniques. It is important to note that the genetic signal from IIL encompasses only unfavorable effects resulting from long IBD segments. As a result, a comparison of the prediction accuracies between the 2 metrics needs to not be interpreted as an exercise of ranking the predictive ability of the 2 metrics (the IIL value would, by construction, capture only a subset of the overall genetic signal) but rather to determine the relationship between complementary metrics, to allow their integration. 


\section{Simulated Data}

Multiple scenarios were simulated to determine the frequency of unfavorable haplotypes being identified by the algorithm. Simulation was performed using Geno-Diver (Howard et al., 2017), a combined coalescence and forward-in-time simulation software. We hypothesized that the amount of short-range linkage disequilibrium (LD) existing in the genome impacts how well the algorithm can identify unfavorable haplotypes. Four scenarios of increasing levels of short-range LD in the historical population were generated as outlined in Supplemental Fig. S1 (see the online version of the article at http://journalofanimalscience.org) and will be referred to as low, low-medium, medium-high, and high, respectively. For each LD scenario, different genetic architectures were simulated with 250,500 , or 1,000 QTL spread equally across 5 chromosomes. The combination of variable LD and QTL parameters produced 12 different scenarios. Each scenario was replicated 25 times.

Within each LD setting, SNP sequence data for 4,000 base haplotypes across 5 chromosomes, each with a length of $150 \mathrm{Mb}$, were simulated by internally calling MaCS (Chen et al., 2009) within the Geno-Diver software. Scenarios with the same LD parameter were initialized using the same set of sequence data to limit the computational time and variability across replicates due to historical sequence information. Following the generation of sequence data, QTL were randomly placed along the genome and a SNP panel with neutral markers was created. A total of 4,000 markers $(20,000$ genomewide) were used within each chromosome. This marker density was chosen to generate a density within each chromosome that is similar to a medium-density marker array such as the Illumina PorcineSNP60K (Illumina Inc., San Diego, CA). Across all scenarios, the minimum minor allele frequency was set at 0.10 and 0.015 for markers and QTL, respectively.

For each QTL the additive effect ( $a$ ) of a QTL, defined as half the difference in genotypic value between the homozygote genotypes (Falconer and Mackay, 1996), was sampled from a gamma distribution (shape $=0.4$ and scale $=1.66)$ with an equal chance of being positive or negative. The dominance effect $(d)$ of a QTL, defined as the deviation of the genotypic value of the heterozygote from the mean of the genotypic values of the 2 homozygotes (Falconer and Mackay, 1996), was generated similarly to the method of Wellmann and Bennewitz (2012). First, the degree of dominance $(h)$ at $\mathrm{QTL}_{i}$ was sampled from a normal distribution $($ mean $=0.1$ and variance $=$ 0.04) and then the dominance effect at $\mathrm{QTL}_{i}$ was calculated as $d_{i}=h_{i}\left|a_{i}\right|$, in which $\left|a_{i}\right|$ is the absolute value of the additive effect. Across all scenarios, the additive and dominance effects were scaled to generate a narrow$\left(h^{2}\right)$ and broad-sense heritability $\left(H^{2}\right)$ of 0.35 and 0.40 , respectively. The normal distribution parameters used to generate the degree of dominance were used to create a trait that displayed directional dominance along with a majority of the loci displaying partial dominance. The phenotype for an individual $\left(y_{i}\right)$ was generated as

$$
y_{i}=\mu+\sum_{q}^{\text {nOTL }}\left(\gamma_{i} a_{q}+\delta_{i} d_{q}\right)+e_{i},
$$

in which $\mu$ is the general mean, $n \mathrm{QTL}$ is the number of QTL, $\gamma$ is the genotype (i.e., 0 for the homozygote, 1 for the heterozygote, and 2 for the alternative homozygote) for individual $i$ at $\mathrm{QTL}_{q}, a$ is the additive substitution effect for $\mathrm{QTL}_{q}, \delta$ is the dominance genotype (i.e., 1 for heterozygote and 0 for either homozygote) for individual $i$ at $\mathrm{QTL}_{q}, d$ is the dominance effect for QTL $_{q}$, and $e_{i}$ is a normal residual such that $e \sim N[0$, $\left(1-q^{2}\right)$ ]. Summary statistics on the QTL architecture and genetic diversity of the 12 scenarios is outlined in Supplemental Table S1 (see the online version of the article at http://journalofanimalscience.org).

After the founder population and genetic architecture of the trait were generated, a selection scenario mimicking a livestock population was undertaken for 10 generations. A population consisting of 50 males and 600 females was used, with a replacement rate of $20 \%$ for both males and females. Progeny with a high EBV were selected to serve as parents for the next generation, and EBV were generated from an animal model based on pedigree information. A low phenotypic value represented the unfavorable direction for the simulated trait in this case. Animals were mated at random, and 1 progeny was produced for each mating pair. Progeny born from generations 7 to 9 served as the training population to identify unfavorable haplotypes, and progeny from generation 10 served as the validation population. The model used to identify unfavorable haplotypes is outlined below:

$$
y=\mathbf{X b}+\mathbf{Z a}+\mathbf{e},
$$

in which $y$ is the phenotype, $\mathbf{b}$ is a vector of fixed effects, $\mathbf{a}$ is a vector of random additive genetic effects, $\mathbf{e}$ is a vector of random residuals, and $\mathbf{X}$ and $\mathbf{Z}$ are incidence matrices relating $\mathbf{b}$ and $\mathbf{a}$, respectively, to $y$. The only fixed effect was the overall mean. The starting window size was set at $60 \mathrm{SNP}$ and was reduced by 5 SNP until a window size of 20 SNP was reached. Different SNP window sizes were investigated based on the density simulated. Similar results were found in terms of the regions identified, associated effects, and the relationship between IIL and the phenotype or true genetic signal (data not shown). The suggestive phenotypic cutoff in step 1 was declared by randomly sampling 1,000 windows to generate the empirical $t$-statistic distribution. 
To investigate the proportion of true negative $\mathrm{ROH}$ effects the algorithm captured within each replicate, the true effect for any $\mathrm{ROH}$ with a length greater than $1 \mathrm{Mb}$ was calculated. A length of $1 \mathrm{Mb}$ was chosen to provide a range of possible $\mathrm{ROH}$ lengths captured by the algorithm. The true negative and positive $\mathrm{ROH}$ effects were split into quantiles of decreasing and increasing effects, respectively. The algorithm tests only for the unfavorable direction, and therefore, the percentage of true ROH effects the algorithm identified is expected to be higher in the negative direction than in the positive direction. Lastly, using the same $1-\mathrm{Mb}$ $\mathrm{ROH}$ cutoff, statistics on the length of ROH the algorithm identified (or missed) were calculated.

Within each replicate, the IIL was estimated based on haplotypes identified in the training population for individuals in the validation population. The correlation between IIL and the true genotypic value (TGV), true breeding value (TBV), and true dominance deviation (TDD) was also estimated. Additionally, the significance (i.e., $-\log P$-value) of IIL or a genome-wide metric when included as a fixed covariate effect in model [2] was estimated for the validation population. The IIL or genome-wide metric was included as a fixed covariate in the similar model (i.e., no ROH effect included in the model) that was used to identify haplotypes in the training population. Three genome-wide inbreeding metrics were used as comparison including pedigree inbreeding (Henderson, 1976), diagonals of the SNP-bySNP relationship matrix (SNPRM; VanRaden, 2008), or proportion of the markers that were homozygous.

To explore the predictive ability of IIL compared with estimates of the genetic value using whole-genome regression models, a Bayesian ridge regression (BRR) analysis was conducted that included the additive and dominance effect for each SNP. The same training and validation generations that were previously used were also used in the BRR analysis. Marker effects were estimated using the BGLR package in R (Perez and de los Campos, 2014). A total of 55,000 iterations were run, with the first 5,000 discarded as burn-in and a thinning rate of 5 iterations. Across individuals, the EBV, estimated dominance deviation (EDD), and estimated genotypic value (EGV) were generated by multiplying the estimated effect by the associated genotype and summing across all markers. The prediction accuracy for either IIL or EGV was determined in the validation population based on the correlation between phenotype and EGV or IIL, respectively. It was standardized by dividing by the square root of the heritability estimated in the training generation for each replicate (Legarra et al., 2008; Wolc et al., 2011). Correlations between IIL and the EBV, EDD or EGV were also estimated.

\section{Swine Data}

Phenotypic and genotypic data from 2 maternal purebred nucleus selection lines were obtained from Smithfield Premium Genetics (Rose Hill, NC). To determine the algorithm's behavior across different genetic architectures, multiple traits were investigated including litter size, litter viability, and growth rate. Individuals with genotype information from Large White (LW; $n=6,750)$ and Landrace (LR; $n=5,010)$ pigs were used. Animals born before 2012 were used as a training population and animals born in 2013 were used as a validation population. The number of animals across traits is outlined in Table 1. A complete description of the genotype quality control is outlined in Howard et al. (2016). Briefly, genotype data was derived from the Illumina PorcineSNP60K BeadChip (Illumina Inc.; version 1 and 2) and the GGP-Porcine (GeneSeek Inc., a Neogen Co., Lincoln, NE). Multiple quality control measures were conducted and are described by Howard et al. (2016). Genotypes were phased and imputed using Beagle (version 3; Browning and Browning, 2007). After quality control and discarding SNP that were poorly imputed, a total of 39,671 and 41,489 autosomal SNP for LW and LR, respectively, remained.

Seven litter size and mortality traits including number born alive (NBA), total number born (TNB), proportion born dead, average litter birth weight (LBW), preweaning mortality (PWM), number weaned (NW), and average litter wean weight (NWBW) were used in the analysis. The TNB phenotype included NBA and stillborn and mummified piglets. The proportion born dead was calculated as $1-(\mathrm{NBA} / \mathrm{TNB})$. The LBW was calculated as the mean weight of the number of live piglets at processing and litters were processed within $48 \mathrm{~h}$ after birth. Traits that were recorded after birth, including PWM, NW, and NWBW, are impacted by the degree of cross-fostering. Cross-fostering in the current data was similar to previous estimates by Putz et al. (2015) in a related population. To minimize the effect of cross-fostering, only litters having more than $75 \%$ of the birth sow piglets were used in the analysis. After the data edit, 98.0 and $97.7 \%$ of the piglets were nursed by their original birth sow for LW and LR, respectively. The PWM phenotype was calculated as the number of piglets that died after $24 \mathrm{~h}$ and included pigs euthanized at weaning divided by the total number of pigs in the litter after the 24-h cutoff. The NWBW was calculated as the average weight of the number of piglets weaned. All reproductive traits were evaluated as a trait of the biological dam, and model [1] was used. The fixed effects used for each trait are outlined in Table 1.

Two production traits were investigated: $\mathrm{BW}$ at off-test (Weight) and ADG. The ADG metric was measured from birth to off-test. Production traits were 
Table 1. The model used, estimated genetic parameters, and the number of animals across traits for the Landrace (LR) and Large White (LW) population

\begin{tabular}{|c|c|c|c|c|c|c|}
\hline \multirow[b]{2}{*}{ Breed } & \multirow[b]{2}{*}{ Trait $^{1}$} & \multirow[b]{2}{*}{ Fixed effects ${ }^{2}$} & \multicolumn{2}{|c|}{ Genetic parameter $^{3}$} & \multicolumn{2}{|c|}{ Animals (records) } \\
\hline & & & $h^{2}$ & $\overline{r^{2}}$ & Training & Validation \\
\hline \multirow[t]{9}{*}{$\overline{\mathrm{LR}}$} & NBA & Parity and CG & 0.082 & 0.149 & $4,005(9,416)$ & $1,005(1,639)$ \\
\hline & TNB & Parity and CG & 0.082 & 0.149 & $4,003(9,302)$ & $998(1,621)$ \\
\hline & $\mathrm{PD}$ & Parity and CG & 0.085 & 0.156 & $4,003(9,293)$ & $998(1,621)$ \\
\hline & LBW & Parity, CG, and NBA & 0.231 & 0.284 & $3,985(8,648)$ & $988(1,586)$ \\
\hline & PWM & Parity, CG, and Pig24h & 0.115 & 0.131 & $3,504(6,724)$ & $870(1,330)$ \\
\hline & NW & Parity, CG, and Pig24h & 0.102 & 0.120 & $3,465(6,600)$ & $845(1,280)$ \\
\hline & NWBW & Parity, CG, Pig24h, and NW & 0.144 & 0.211 & $3,465(6,600)$ & $845(1,280)$ \\
\hline & Weight & CG, sex, and age & 0.271 & - & $4,386(4,386)$ & $993(993)$ \\
\hline & ADG & CG, sex, and age & 0.271 & - & $4,386(4,386)$ & $993(993)$ \\
\hline \multirow[t]{9}{*}{ LW } & NBA & Parity and CG & 0.115 & 0.150 & $5,518(15,014)$ & $1,232(2,271)$ \\
\hline & TNB & Parity and CG & 0.098 & 0.142 & $5,513(14,673)$ & $1,228(2,262)$ \\
\hline & PD & Parity and CG & 0.086 & 0.133 & $5,513(14,664)$ & $1,228(2,262)$ \\
\hline & LBW & Parity and CG, NBA & 0.241 & 0.307 & $5,487(13,581)$ & $1,188(2,155)$ \\
\hline & PWM & Parity, CG, and Pig24h & 0.074 & 0.168 & $4,966(10,464)$ & $1,102(1,824)$ \\
\hline & NW & Parity, CG, and Pig24h & 0.053 & 0.115 & $4,901(10,259)$ & $1,054(1,716)$ \\
\hline & NWBW & Parity, CG, Pig24h, and NW & 0.144 & 0.203 & $4,901(10,259)$ & $1,054(1,716)$ \\
\hline & Weight & CG, sex, and age & 0.292 & - & $5,576(5,576)$ & $1,197(1,197)$ \\
\hline & ADG & CG, sex, and age & 0.291 & - & $5,576(5,576)$ & $1,197(1,197)$ \\
\hline
\end{tabular}

${ }^{1} \mathrm{NBA}=$ number born alive; TNB = total number born; $\mathrm{PD}=$ proportion born dead; $\mathrm{LBW}=$ average litter birth weight; $\mathrm{PWM}=$ preweaning mortality; $\mathrm{NW}=$ number weaned; NWBW $=$ average litter wean weight; weight represents weight at off-test.

${ }^{2} \mathrm{CG}=$ contemporary group (based on farm, year, and season); Pig24h = pigs in the litter after the 24-h cutoff; age refers to off-test age.

${ }^{3} h^{2}$ refers to the narrow sense heritability; $r^{2}$ refers to the repeatability.

evaluated as a trait of the animal, and model [2] was used. The fixed effects used for each trait are described in Table 1. Across both reproductive and production traits, the contemporary group was composed of farm, year, and season, and any animal that was within a contemporary group smaller than 5 animals was removed from the analysis.

Summary statistics on the length of $\mathrm{ROH}$ and the unfavorable haplotypes captured were generated. Prediction accuracy for IIL was compared with a whole-genome regression BRR model. For all 9 traits, yield deviations were constructed for each trait based on the fixed effects outlined in Table 1. For the reproductive traits, an animal may have multiple observations, and therefore, average yield deviations were used and the residuals for a given observation in the BRR analysis were weighted according to Garrick et al. (2009). The formula used to calculate the weight was

$$
\left(1-h^{2}\right) /\left(h^{2}+\left\{\left[1+r^{2}(l-1)\right] / l\right\}-h^{2},\right.
$$

in which $h^{2}$ refers to the heritability, $r^{2}$ refers to the repeatability, and $l$ refers to the number of records. The values used for $h^{2}$ and $r^{2}$ are outlined in Table 1 across all 9 traits. Using BGLR (Perez and de los Campos, 2014), a total of 155,000 iterations were run, with the first 5,000 discarded as burn-in and a thinning rate of 5 iterations. Again, for each trait, the EGV values were predicted.
The accuracy of predicting the phenotype using IIL compared with a whole-genome regression model was investigated. Also, the relationship between IIL and values derived from a whole-genome regression model was investigated (EGV, EBV and EDD). The prediction accuracy for either IIL or EGV across the 9 traits was determined in the validation population. The prediction accuracy was calculated as the correlation between the IIL or EGV and average yield deviation. It was standardized by dividing it by the square root of the heritability for each trait. The significance of the IIL or genome-wide regression coefficient was estimated as previously outlined.

Lastly, the correlation between the diagonal and the off-diagonal elements of the ILM across traits and with pedigree- and genomic-based relationship matrices was estimated. Understanding the correlation between ILM across traits is important when an ILM is used to minimize inbreeding depression across all traits in a breeding objective. Any change in the off-diagonal ILM value for one trait should ideally result in a favorable or negligible change in the off-diagonal ILM value for other traits. The ILM was compared with 3 relationship matrices including a pedigree-based matrix (A; Henderson, 1976), the SNPRM, and a ROH-based relationship matrix with a 5-Mb cutoff (ROH5RM; Howard et al., 2016). To determine the sensitivity of fixing the variance components based on the null model of no ROH effect, the ASReml program (Gilmour et 

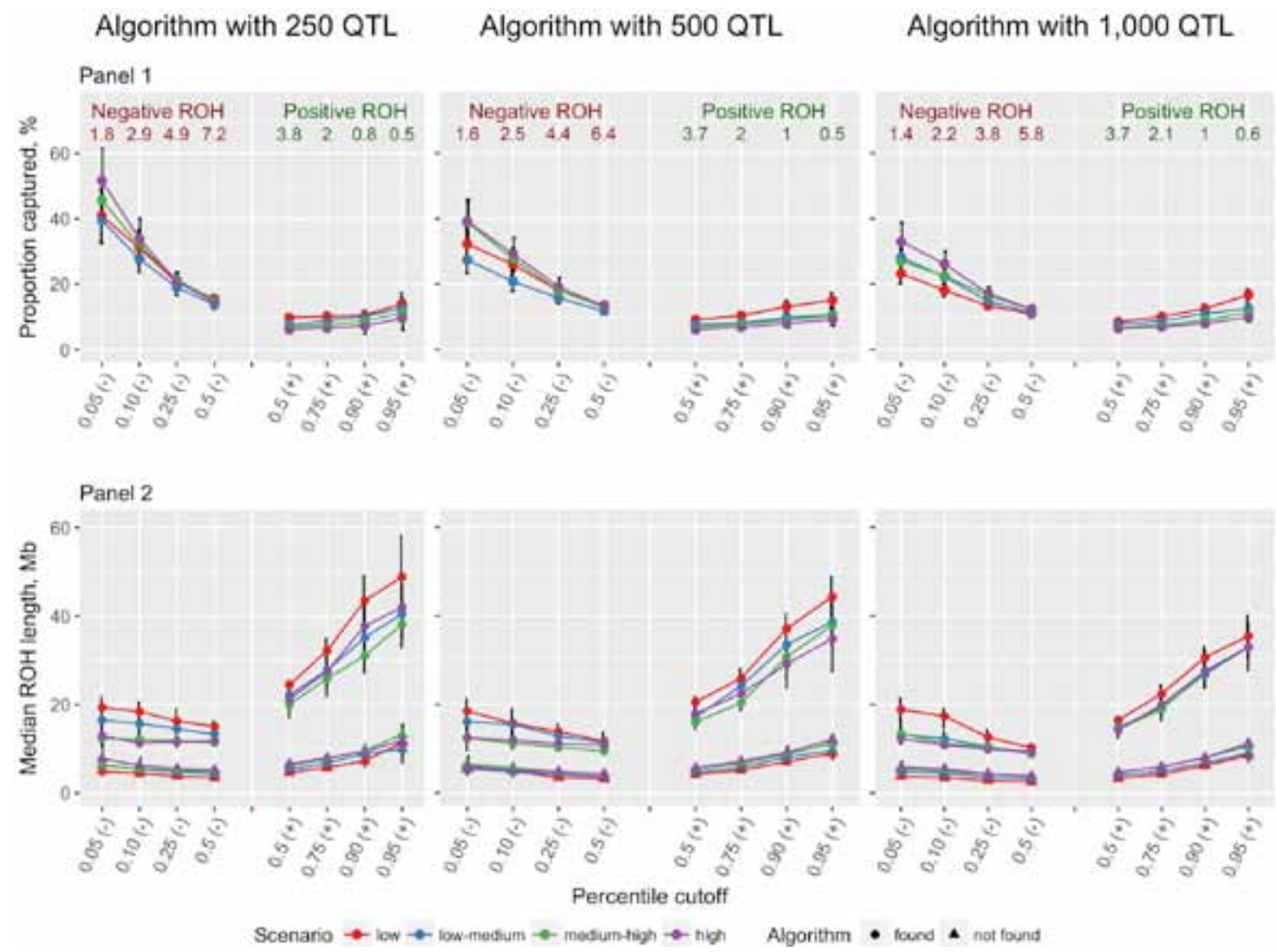

Figure 2. Summary of the proportion of runs of homozygosity (ROH) of at least $1 \mathrm{Mb}$ the algorithm captures (panel 1) and the length of the ROH the haplotype tags (panel 2) across simulation scenarios by percentile class and whether the algorithm identified the haplotype. In panel 1 , the summary statistic is the mean and $95 \%$ confidence interval, and in panel 2 , the summary statistic is the first, second and third quartiles. The simulation scenarios refer to the ancestral population scenario simulated. Scenario 1 uses the "Ne1000" parameter in Geno-Diver (Howard et al., 2017), scenario 2 uses the "Ne250" parameter in Geno-Diver, scenario 3 uses the "Ne100_Scen1" parameter in Geno-Diver, and scenario 4 uses the "Ne70" parameter in Geno-Diver. The $\mathrm{ROH}$ percentile class is outlined on the $x$-axis; the first 4 percentile classes represent quantiles for the true negative effects (i.e., 0.05 represents highly negative ROH effects) and the last 4 percentile classes represent quantiles for the true positive effects (i.e., 0.95 represents highly positive effects). The $y$-axis shows to the algorithms summary statistics for the number of true ROH identified out of all true ROH effects within the given quintile. The number below negative or positive $\mathrm{ROH}$ refers to the percentage of the $\mathrm{ROH}$ that the algorithm correctly found out of the all negative or positive true ROH effects.

al., 2009), which reestimates variance components for each window, was used across breed and traits for the windows that were deemed significant by the algorithm.

\section{RESULTS}

\section{Simulated Data}

A summary of how effectively the algorithm identified true negative and positive $\mathrm{ROH}$ effects across different percentiles is outlined in panel 1 of Fig. 2. Because the algorithm tests for only the unfavorable direction, the percentage of true $\mathrm{ROH}$ effects the method identifies is expected to be greater than 0 in the negative direction and 0 in the positive direction. As illustrated in panel 1 of Fig. 2, as the true negative unfavorable ROH effect got larger, a greater proportion of unfavorable $\mathrm{ROH}$ genotypes was identified by the algorithm. It should be noted that, averaged across all scenarios, the frequency of highly unfavorable ROH effects was small (1.8\%) compared with the total number of true negative $\mathrm{ROH}$ effects. The frequency of incorrectly identified positive $\mathrm{ROH}$ effects (i.e., false positives) by the algorithm remained relatively flat across all percentiles and was, on average, 9.4\% (95\% confidence interval [CI] 8.7-10.1) across all scenarios. As the LD in the population increased and became similar to that of most livestock populations, the algorithm was more effective at identifying unfavorable haplotypes and had a lower false positive rate. For example, for true $\mathrm{ROH}$ effects with the largest negative effect (i.e., less than the 0.05 percentile), the algorithm identified, on average, 32.1 (95\% CI 28.5$35.7)$ and $41.2 \%$ (95\% CI 36.7-45.9) of the total true negative ROH effects across the 3 QTL scenarios for the low- and high-LD scenarios, respectively. Conversely, for incorrectly identified true ROH effects (i.e., estimated to be negative but had a true positive effect) with the largest positive effect (i.e., greater than the 0.95 percentile), the algorithm identified, on average, 15.3 (95\% CI $13.9-16.7)$ and $9.6 \%(95 \%$ CI 8.2-10.9) of the total true ROH effects across the 3 QTL scenarios for the low- and high-LD scenario, respectively. 
Summary statistics on the length of $\mathrm{ROH}$ of at least $1 \mathrm{Mb}$ tagged by the unfavorable haplotype is outlined in panel 2 of Fig. 2. We report the median in this case, rather the mean, because the distribution of the length of $\mathrm{ROH}$ containing a tag haplotype has a heavy tail and, therefore, the latter parameter is heavily influenced by extreme values. The length of $\mathrm{ROH}$ tagged by the identified haplotypes for the medium-high and high LD scenarios was similar across negative percentiles and QTL scenarios with a median (first quartile- third quartile) of $12.15 \mathrm{Mb}$ (10.07-13.41). The haplotypes identified for the low and low-medium LD scenarios across negative percentiles and QTL tagged longer ROH stretches, with a median length of $15.77 \mathrm{Mb}(12.23-18.64)$. The results show how the core unfavorable haplotype identified by the algorithm, which had a median length of $7.0 \mathrm{~kb}$ across scenarios, in reality serves as a proxy for a much larger observed $\mathrm{ROH}$ segment. The length of unfavorable ROH that the algorithm missed was made of considerably smaller ROH (median [first quartilethird quartile]: $5.26 \mathrm{Mb}$ [4.06-5.81]) and was again similar across negative percentiles and scenarios. For the incorrectly identified true positive $\mathrm{ROH}$ effects, it should be noted that the length of ROH captured by the haplotype gets longer proportional to the true $\mathrm{ROH}$ effect. Therefore, in general, falsely identified $\mathrm{ROH}$ regions were, in our analysis, characterized by being locally negative around the identified unfavorable haplotype. Yet as a result of being part of an extremely large $\mathrm{ROH}$, positive QTL effects contained in the long $\mathrm{ROH}$ genotype made the overall effect positive.

The relationship of IIL with the true genetic signal, the predictive ability of IIL compared with wholegenome regression values, and the significance of IIL or genome-wide inbreeding regression coefficients are outlined in Fig. 3. Panel 1 of Fig. 3 describes the correlation between IIL and TGV, TBV, and TDD. Across all QTL scenarios, the correlation increased as the LD increased for all parameters except TDD. Averaged across QTL scenarios, the correlation between IIL and the TGV for the low- and high-LD scenario was 0.31 (95\% CI 0.29 0.32 ) and 0.44 (95\% CI $0.42-0.45)$, respectively. The correlation between IIL and TBV was similar to the correlation between IIL and TGV. The average correlation between IIL and TDD was 0.002 (95\% CI -0.01 to 0.01 ) for all scenarios, which was not unexpected, given the fact that the $\mathrm{ROH}$ effects are a function of the alternative homozygote genotypes and not heterozygous genotypes.

Panels 2 and 3 of Fig. 3 summarize the effectiveness of using the IIL algorithm based on its predictive ability or as a tool to minimize the frequency of unfavorable haplotypes in the progeny. As outlined in panel 2 of Fig. 3, the correlation between IIL and the phenotype increased as the level of LD increased in the population.
Averaged across QTL scenarios, the prediction accuracy of IIL was 0.34 (95\% CI $0.32-0.36)$ and 0.49 (95\% CI $0.47-0.52$ ) for the low- and high-LD scenarios, respectively. Similar trends of increasing prediction accuracy as LD increased in a population were seen for the wholegenome prediction values. As well, minor differences were found between the prediction accuracy for EGV and EBV. Averaged across QTL scenarios, the prediction accuracy of EGV was 0.66 (95\% CI $0.64-0.67)$ and 0.82 (95\% CI 0.79-0.84) for the low- and high-LD scenarios, respectively. These results are not unexpected because the algorithm uses haplotypes that have only an unfavorable effect contained within $\mathrm{ROH}$ stretches and favorable haplotypes are not included in IIL. The correlations between IIL and values from the whole-genome regression model are outlined in Supplemental Fig. S2, panel 1 (see the online version of the article at http://journalofanimalscience.org). Averaged across scenarios, the correlation between IIL and EGV was 0.50 (95\% CI 0.49-0.50), and in general, as the LD increased, so did the correlation.

The last summary statistic in panel 3 of Fig. 3 outlines the significance of the regression coefficient based on either genome-wide inbreeding metrics or IIL. Across all genome-wide inbreeding metrics, the $-\log$ $P$-value was similar across all LD scenarios and the significance increased in proportion to the number of QTL. For example, averaged across scenarios and genomewide inbreeding metrics, the average $-\log P$-values were $1.12(95 \%$ CI $1.01-1.23)$ and $1.68(95 \%$ CI 1.49-1.87) for the scenarios with 250 and 1,000 QTL, respectively. The $-\log P$-value for the IIL metric across all scenarios was, in all cases, greater and increased as the LD in the population increased. Under the high-LD scenario, the average $-\log P$-value for the IIL metric across QTL scenarios was 21.24 (95\% CI 19.37-23.10), corresponding to a nominal $P$-value of $5.96 \times 10^{-10}$.

In summary, the simulation results highlight that the algorithm identified, on average, $41 \%$ of the highly unfavorable (i.e., 0.05 percentile) $\mathrm{ROH}$ effects across the QTL scenarios and under the high-LD scenario. Moreover, the unfavorable haplotypes were effective at tagging a significantly larger $\mathrm{ROH}$ region. Under the high-LD scenario, which closely resembles most livestock situations, the $\mathrm{ROH}$ that the haplotype tagged had a median length of $12.1 \mathrm{Mb}$. When combining all unfavorable haplotypes based on their probability of occurring and the effect of the haplotype being in a ROH, a moderate prediction accuracy was achieved. Furthermore, the correlation between IIL and the EGV was moderate or, more importantly, less than unity in all cases. Therefore, a combination of IIL and a genomewide genetic value would allow for 2 animals with similar genetic values but a different number of unfavorable haplotypes contained within long $\mathrm{ROH}$ to be 

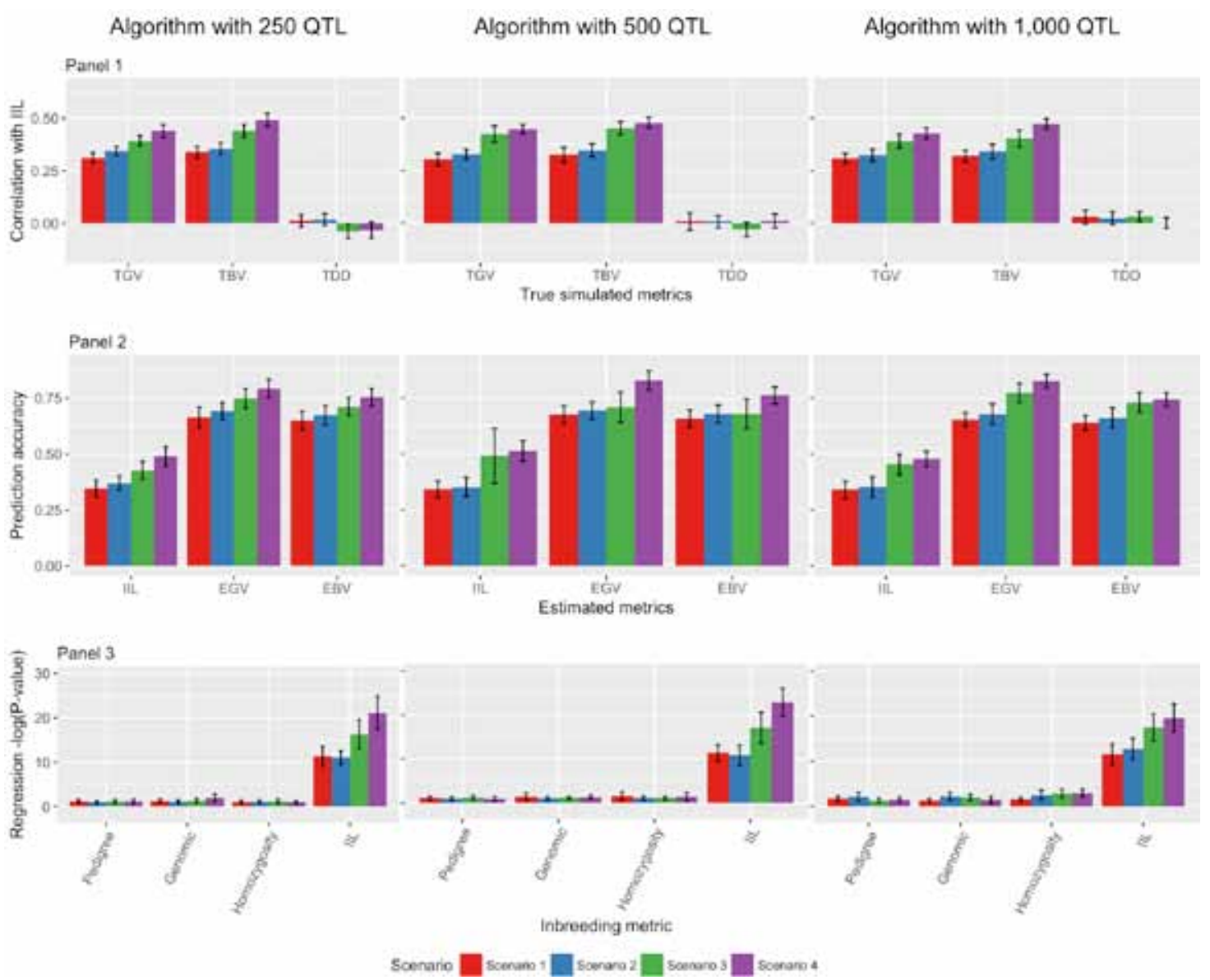

Figure 3. Summary (mean and $95 \%$ confidence interval) of the correlation between individual inbreeding load (IIL) and the true genetic signal (panel 1), prediction accuracy for IIL estimates from a Bayesian ridge regression analysis (panel 2), and significance of the regression coefficient based on either a genome-wide inbreeding metrics or IIL (panel 3) across simulation scenarios. TGV = true genotypic value; TBV = true breeding value; TDD = true dominance deviation; EGV = estimated genotypic value. In panel 3, "Pedigree" refers to pedigree inbreeding, "Genomic" refers to genomic inbreeding based on diagonals of genomic relationship matrix, and "Homozygosity" refers to the proportion of genome that is homozygous. The simulation scenarios refer to the ancestral population scenario simulated. Scenario 1 uses the "Ne1000" parameter in Geno-Diver (Howard et al., 2017), scenario 2 uses the "Ne250" parameter in Geno-Diver, scenario 3 uses the "Ne100 Scen1" parameter in Geno-Diver, and scenario 4 uses the "Ne70" parameter in Geno-Diver.

distinguished. The running time for the algorithm averaged across all 12 simulation scenarios was 40.75 min. The computations were done on a Dell Precision T3500 with 2 Intel Xeon X5482 3.20 GHz processors and 24 GB of RAM using 2 threads.

\section{Swine Data}

To determine whether similar results were found with real data and to investigate its effectiveness across multiple traits, the algorithm was tested with 2 swine commercial maternal lines. The significance of the regression coefficient and the predictive ability of IIL compared with genome-wide inbreeding metrics is presented in Table 2. Across both breeds and for the majority of traits except for NBA and TNB in LR, IIL had a prediction accuracy greater than 0 . Averaged across traits within a breed, the average prediction accuracy was 0.15 (SD 0.13) and 0.20 (SD 0.04) for LR and LW, respectively. Similar to the simulation, the whole-genome regression-based EGV resulted in higher prediction accuracies compared with IIL across all traits and breed. The prediction accuracy averaged across traits within a breed was 0.48 (SD 0.10) and 0.49 (SD 0.17) for LR and $\mathrm{LW}$, respectively. Both prediction accuracies were lower than what was achieved in the simulation, given the lower heritability for most of the traits and the simplified assumptions used in the simulation. The correlations between IIL and values from the whole-genome regression model are outlined in the bottom of Supplemental Fig. S2, panel 2 (see the online version of the article at http:// journalofanimalscience.org). Averaged across traits within a breed, the correlations between the IIL and EGV were 0.31 (SD 0.13) and 0.32 (SD 0.06) for LR and LW, respectively. A positive correlation (average for LR was 0.07 [SD 0.06] and for LW was 0.15 [SD 0.07]) was estimated between IIL and EDD.

Also outlined in Table 2 is the $-\log P$-value of the regression coefficient when genome-wide inbreeding or IIL values were included in the model. Averaged across 
traits within a breed, the IIL regression coefficient resulted in a higher $-\log P$-value (i.e., lower $P$-value) across both breeds compared with any genome-wide inbreeding metric, whereas the pedigree-based inbreeding metric had the lowest $-\log P$-value. Out of the 9 traits, the regression coefficient was trending toward significance $(P$-value $<0.10)$ for 6 (LBW, PWM, NW, NWBW, weight, and ADG) and 7 (NBA, TNB, LBW, NW, NWBW, weight, and ADG) out of the 9 traits for LR and LW, respectively. Alternatively, for genomewide inbreeding metrics, the regression coefficient for the proportion of the genome homozygous, diagonals of SNPRM, or pedigree-based inbreeding was trending toward significance for 3, 2, and 0 traits, respectively, for LR and 4, 2, and 0 traits, respectively, for LW. Therefore, in our results, IIL was the parameter that more closely aligned with the identification of functional inbreeding. It should be noted that no single parameter had a consistently higher $-\log P$-value across traits, so a combination of genome-wide inbreeding metric based on genomic information and the IIL value would likely be optimal in breeding applications.

An ideogram of regions of the genome where an unfavorable haplotype was identified by the algorithm across the 9 traits for the 2 lines is depicted in Supplemental Fig. S3 and S4 (see the online version of the article at http://journalofanimalscience.org). Regions of the genome with long unfavorable stretches of homozygosity were observed across multiple traits/ line. Conversely, other regions did not appear to harbor unfavorable stretches of homozygosity. The number of regions that have an unfavorable effect across at least 4 of the 9 traits is outlined in Table 3 and regions were placed into categories based on the relationship between the traits. A summary of the regions and the least squares mean difference between an animal in a $\mathrm{ROH}$ versus non- $\mathrm{ROH}$ across both breeds is outlined in Supplemental Table S2 (see the online version of the article at http://journalofanimalscience.org). A total of 4 and 13 regions were found that had at least 1 production and reproduction trait associated with a unfavorable haplotype in LR and LW, respectively. A total of 3 regions across both breeds were associated with only reproductive traits. Summary statistics on the median $\mathrm{ROH}$ length that the unfavorable haplotype tagged and the average frequency of the ROH genotype across traits and breeds is outlined in Supplemental Table S3 (see the online version of the article at http://journalofanimalscience.org). The average median length of the unfavorable haplotype across trait and breeds was 1.56 and $1.54 \mathrm{Mb}$ for LR and LW, respectively. Similarly to what was found in the simulated data, the unfavorable haplotype tagged a larger ROH of 9.55 and $9.12 \mathrm{Mb}$ averaged across traits within LR and LW, respectively,
Table 2. The significance of inbreeding regression coefficient across multiple inbreeding metrics and the prediction accuracy of the individual inbreeding load (IIL) and estimated genotypic value (EGV) from whole-genome Bayesian ridge regression across traits for Landrace (LR) and Large White (LW) populations

\begin{tabular}{|c|c|c|c|c|c|c|c|}
\hline \multirow[b]{2}{*}{ Breed } & \multirow[b]{2}{*}{ Trait $^{1}$} & \multicolumn{4}{|c|}{$\begin{array}{c}\text { Regression on adjusted } \\
\text { phenotype }-\log (P \text {-value })^{2}\end{array}$} & \multicolumn{2}{|c|}{$\begin{array}{c}\text { Prediction } \\
\text { accuracy }\end{array}$} \\
\hline & & $\begin{array}{l}\text { Pedigree } \\
\text { inbreeding }\end{array}$ & $\begin{array}{l}\text { Genomic } \\
\text { inbreeding }\end{array}$ & $\begin{array}{l}\text { Proportion } \\
\text { homozygous }\end{array}$ & IIL & EGV & IIL \\
\hline \multirow[t]{10}{*}{ LR } & NBA & 0.66 & 0.49 & 0.69 & 0.00 & 0.44 & -0.05 \\
\hline & $\mathrm{TNB}$ & 0.87 & 0.68 & 0.23 & 0.53 & 0.42 & -0.09 \\
\hline & PD & 0.15 & 0.00 & 1.42 & 1.94 & 0.53 & 0.15 \\
\hline & LBW & 0.45 & 2.24 & 1.34 & 15.24 & 0.65 & 0.33 \\
\hline & PWM & 0.29 & 1.54 & 0.8 & 2.94 & 0.40 & 0.17 \\
\hline & NW & 0.21 & 1.01 & 0.49 & 4.00 & 0.42 & 0.22 \\
\hline & NWBW & 2.14 & 0.66 & 4.36 & 2.51 & 0.64 & 0.22 \\
\hline & Weight & 0.53 & 4.52 & 2.93 & 3.24 & 0.40 & 0.17 \\
\hline & $\mathrm{ADG}$ & 0.58 & 4.54 & 2.84 & 4.00 & 0.39 & 0.18 \\
\hline & Average & 0.65 & 1.74 & 1.68 & 3.82 & 0.48 & 0.15 \\
\hline \multirow[t]{10}{*}{ LW } & NBA & 0.62 & 0.15 & 0.12 & 4.14 & 0.45 & 0.24 \\
\hline & TNB & 0.98 & 0.40 & 0.21 & 2.56 & 0.42 & 0.19 \\
\hline & PD & 0.54 & 1.35 & 1.97 & 1.94 & 0.38 & 0.13 \\
\hline & LBW & 2.18 & 1.11 & 4.07 & 10.25 & 0.77 & 0.24 \\
\hline & PWM & 2.11 & 0.33 & 1.37 & 2.09 & 0.36 & 0.18 \\
\hline & NW & 1.71 & 0.12 & 0.00 & 2.91 & 0.29 & 0.25 \\
\hline & NWBW & 0.00 & 0.78 & 2.56 & 3.46 & 0.79 & 0.17 \\
\hline & Weight & 0.69 & 5.70 & 6.14 & 5.99 & 0.48 & 0.18 \\
\hline & $\mathrm{ADG}$ & 0.73 & 5.76 & 6.09 & 4.77 & 0.48 & 0.17 \\
\hline & Average & 1.06 & 1.74 & 2.50 & 4.23 & 0.49 & 0.20 \\
\hline
\end{tabular}

${ }^{1} \mathrm{NBA}=$ number born alive; $\mathrm{TNB}=$ total number born; $\mathrm{PD}=$ proportion born dead; LBW = average litter birth weight; PWM = preweaning mortality; NW = number weaned; NWBW = average litter wean weight; weight represents weight at off-test.

${ }^{2}$ As a reference, a $P$-value of $0.10,0.05$, and 0.01 is equivalent to a negative $\log P$-value of $2.30,3.0$, and 4.6 , respectively.

corresponding to (averaged across traits) 172 and 156 SNP for LR and LW, respectively.

Lastly, the correlation between the diagonals and off-diagonals of the ILM for each trait and genome-wide relationship matrices is presented in Supplemental Fig. S5 and S6 (see the online version of the article at http:// journalofanimalscience.org) for LR and LW, respectively. As shown by the lower diagonal of each matrix, correlations between the off-diagonal elements of the ILM across all traits and genome-wide relationships are all favorably correlated. Any change in the off-diagonal ILM value for one trait would result in a similar (in the favorable direction) or negligible change in the other traits. The average off-diagonal elements across traits for LR had an absolute correlation of $0.23,0.28$, and 0.34 for A, SNPRM, and ROH5RM, respectively. Slightly lower correlations were found for LW, and averaged across traits, the absolute correlation was $0.14,0.20$, and 0.21 for A, SNPRM, and ROH5RM, respectively. In general, 
Table 3. Summary of the number of haplotypes that displayed unfavorable effects across multiple (i.e., $>4$ ) traits for Landrace (LR) and Large White (LW) populations

\begin{tabular}{llc}
\hline \hline Breed & \multicolumn{1}{c}{ Type of trait ${ }^{1}$} & Number of haplotypes \\
\hline LR & Production and reproduction & 4 \\
& Reproduction & 3 \\
\multirow{2}{*}{ LW } & Production and reproduction & 13 \\
& Reproduction & 3 \\
\hline
\end{tabular}

${ }^{1}$ The type of trait refers to a production trait (i.e., weight at off-test and average daily BW gain from birth to off-test) or a reproductive trait (i.e., number born alive, total number born, proportion born dead, average litter birth weight, preweaning mortality, number weaned, and average litter wean weight).

the correlations between the IIL values across traits and genome-wide inbreeding metrics were similar to the off-diagonals and the majority of them were in the same direction. In some instances, the correlations between the values were antagonistic. For example, the SNPRMbased genome-wide inbreeding and IIL values for LR across all traits were antagonistic, although the correlations between the genome-wide inbreeding metrics were much lower compared with the off-diagonal elements.

\section{DISCUSSION}

The objective of this study was to implement a strategy to identify haplotypes within long $\mathrm{ROH}$ that tag an IBD segment due to recent inbreeding. Haplotypes within $\mathrm{ROH}$ were targeted because previous results obtained through simulation by Keller et al. (2011) have shown that $\mathrm{ROH}$-based genome-wide inbreeding metrics have a higher association with the recessive mutation load compared with pedigree- or SNP-by-SNP-based inbreeding metrics. The rationale behind the proposed algorithm stems from previous research investigating the phenotypic effect of a region being in a ROH (Pryce et al., 2014; Howard et al., 2015; Saura et al., 2015). One of the major pitfalls of previously used methods is that they assume that any $\mathrm{ROH}$ genotype within a region of interest has an unfavorable effect, which is most likely not the case. Instead, the unfavorable effect is likely due to a single unique $\mathrm{ROH}$ genotype, with the remaining ones resulting in no unfavorable effect. As a result, the identification of the unique $\mathrm{ROH}$ genotype associated with an unfavorable phenotype allows for the region to be more effectively managed across time. The primary outcome of the proposed algorithm is a list of unfavorable haplotypes. Multiple algorithms already exist to manage unfavorable mutations or haplotypes within breeding programs so that the ones identified by the algorithm could be easily incorporated into previously developed pipelines (Kinghorn, 2011; Cole, 2015).

Within the algorithm, multiple aggregation steps are implemented to confine the unfavorable haplotype to the core of the observed $\mathrm{ROH}$ genotype in a way that is consistent across individuals. As result of the aggregation step, each haplotype serves as a tag for a much larger ROH segment. In this regard, the data presented confirm that the aggregation steps are successful in identifying tag haplotypes contained within a much larger $\mathrm{ROH}$ genotype. Across both swine breeds and in the simulated data set, the median length of the $\mathrm{ROH}$ the haplotype tagged was greater than $9 \mathrm{Mb}$ and the tag haplotype was around $1 \mathrm{Mb}$. Furthermore, simulation results highlighted that the true $\mathrm{ROH}$ effects that were not identified were shorter ROH $(5.26 \mathrm{Mb})$ than the ones that were identified $(13.96 \mathrm{Mb})$. The ability to capture short IBD regions depends on the marker density as described by Ferenčaković et al. (2013), and the marker density used in the current study might not be sufficient to effectively capture these short IBD regions. The impact of the density was not investigated here to limit the number of scenarios generated, yet its impact should be considered in the future. Lastly, the simulation highlighted how, in some cases, the algorithm incorrectly identified true positive $\mathrm{ROH}$ effects that were characterized as being much longer than correctly identified negative $\mathrm{ROH}$ effects. The distribution of the length of ROH has a heavy tail, and therefore, the frequency of long ROH is low, but long ROH do exist within the genome across individuals. These incorrectly identified true positive $\mathrm{ROH}$ regions were locally negative around the tagged unfavorable haplotype, but because they were longer than average $\mathrm{ROH}$, their combined effect was ultimately positive.

We investigated the ability of the algorithm to identify unfavorable haplotypes and their potential use. The frequency at which ROH occur within the genome had a large impact on the ability of the algorithm to identify unfavorable haplotypes. Medium-high and high LD scenarios have LD patterns similar to those observed in livestock species. Under these premises, the algorithm was effective at capturing unfavorable genomic regions. The proportion of highly unfavorable $\mathrm{ROH}$ genotypes (i.e., $<0.05$ percentile) that the algorithm captured under the high-LD scenario varied across QTL scenarios. As the number of QTL increased, the proportion of $\mathrm{ROH}$ genotypes captured decreased. The average proportion of highly unfavorable $\mathrm{ROH}$ genotypes the algorithm captured was 0.52 (95\% CI $0.42-0.62), 0.39$ (95\% CI $0.32-0.46)$, and $0.33 \%(95 \%$ CI $0.27-0.39)$ for the scenarios with 250, 500, and 1,000 QTL, respectively. The prediction accuracy based on real data (average 0.17 [SD 0.10]) was roughly half of what was observed with the simulated data (mean of 0.49 [95\% CI $0.47-0.52$ ] in the high-LD scenario), although across the majority of traits, IIL had a prediction accuracy that was greater than 0 . A prediction accuracy near 0 was observed in LR 
for NBA and TNB, which may be due to multiple factors including purging of unfavorable $\mathrm{ROH}$ genotypes due to strong selection for initial litter size within the line as well as a smaller data set than the one used in the LW population. In our study, whole-genome regressionbased EGV resulted in a moderate predictive ability (average across trait and breed: 0.48 [SD 0.14]). The use of a whole-genome regression method to benchmark the algorithm was used to illustrate the limitations of the algorithm. The algorithm tests for only regions contained in longer $\mathrm{ROH}$ resulting in an unfavorable phenotype and should be used in conjunction with other methods to increase the overall genomic variability and limit the accumulation of inbreeding. Importantly, a moderate positive correlation between IIL and EGV was observed in the simulated (mean of 0.54 [ $95 \%$ CI $0.53-0.56$ ] in the high-LD scenario) and swine (average 0.31 [SD 0.10]) data sets. Therefore, the combination of the 2 metrics could allow for a breeder to more effectively manage the risks associated with sire or mate selection. As a result, a breeder is able to more effectively evaluate the tradeoff between the genetic value of the progeny and undesirable side effects associated with inbreeding. Lastly, the use of the algorithm along with methods to identify lethal mutations/haplotypes (VanRaden et al., 2011) would allow breeders to comprehensively manage genomic diversity and recessive load in a population.

The 2 maternal lines used in this study have been under intense selection for multiple generations, which has potentially resulted in high and heterogeneous levels of homozygosity across the genome. This has been recently investigated by Howard et al. (2016), who estimated the proportion of the genome in a $\mathrm{ROH}$ of at least $5 \mathrm{Mb}$ to be 0.17 and $0.19 \%$ for LR and LW, respectively. Furthermore, nearly all chromosomes across both breeds contained regions of the genome with high levels of ROH. Under this premise, it is likely that the impact of genome-level homozygosity would be regressed toward 0 , because homozygosity in some regions of the genome would no longer be unfavorable. This result is partially verified by the ideogram outlined in Supplemental Fig. S3 and S4 (see the online version of the article at http://journalofanimalscience.org), whereby some regions of the genome have unfavorable haplotypes spread across multiple traits and other do not have any unfavorable regions. The impact of haplotypes contained within a $\mathrm{ROH}$ for regions that were significant across multiple traits can be quite large. For example, an animal homozygous for a tag haplotype on SSC9 (28.9-30.6) within the LR breed would be predicted to have 1.66 fewer pigs born alive, 1.32 fewer total pigs born, and $4.0 \%$ more pigs born dead and the litter would be, on average, $0.07 \mathrm{~kg}$ smaller than an animal that was not homozygous for the tag haplotype.
In general, the genetic diversity of a population is managed through the relationship of the parents based on the expectation that the inbreeding in the progeny is equal to half of the coancestry between the parents (Falconer and Mackay, 1996). As previously discussed, because inbreeding depression is heterogeneous across the genome, a measure that has a higher relationship with the genetic load of an individual may serve as a better metric to manage the degree of inbreeding depression that exists within a population. Therefore, linear mixed models (i.e., model [1] or model [2] outlined in the Materials and Methods section) that included either genome-wide inbreeding metrics or the IIL value in predicting a phenotype were evaluated and the corresponding $-\log P$-value was estimated for each specific inbreeding regression coefficient. Across all simulated scenarios and, on average, across both swine breeds, the significance of the regression coefficient for the IIL value was higher compared with any other genomewide metric. Yet for some traits, genome-wide metrics were more significant. When both the genome-wide inbreeding metric with the highest significance and the IIL were included in the model, similar significance values remained. This highlights how genome-wide inbreeding and IIL metrics are capturing different signals. Based on our results, a combination of a genome-wide relationship matrix and an ILM could be useful in effectively managing the risks associated with choosing an individual/mating combination. Future research should look at the long-term benefits of including the ILM in mating designs in terms of diversity and genetic load. Also, methods to incorporate multiple metrics including the genetic value, genetic diversity, lethal mutations, and the unfavorable haplotypes from the algorithm into an index value should be developed.

Breeding objectives are, in the near totality of cases, composed of several economically important traits, and therefore, the relationship between the ILM across traits is of importance. For the 2 breeds investigated, the offdiagonal values across all traits resulted in a favorable or negligible change across all traits. Therefore, the use of the ILM for a given trait would result in a favorable increase or negligible change in the phenotype of the remaining traits. Furthermore, based on genome-wide relationship metrics (i.e., pedigree or genomic), the off-diagonal elements are favorably correlated with offdiagonal elements of the ILM across traits and breeds. Therefore, as one changes the ILM values in the favorable direction, the relationship across mating pairs is reduced, which is desirable and expected. Future research should investigate methods to combine ILM across traits in the breeding objective. In general, the diagonal values had trends similar to the off-diagonal values across traits and relationship matrices. One of the major differences 
between the 2 values was related to an antagonistic relationship for LR between diagonals of SNPRM and the IIL values across all traits. The inbreeding correlations had a much lower correlation than off-diagonals elements and even more so within the LR breed.

In the present study, variance components were not reestimated for each window in Stage 2, which may have impacted the $t$-statistic. We used ASReml, which does reestimate the variance components for each window, to determine the sensitivity of fixing variance components. The difference between the $t$-statistic from the algorithm and the one from ASReml is outlined in Supplemental Table S4 (see the online version of the article at http:// journalofanimalscience.org). Across all breeds and traits, the differences between the 2 were negligible. In addition, across all breeds and traits, the $t$-statistic from the algorithm gave a conservative estimate compared with the ones derived from ASReml. The proposed algorithm may tag short haplotypes that have a lower probability of being true IBD segments than long ones. This can be minimized with the use of sequence information, which has greater power to correctly identify short IBD segments of the genome (Bosse et al., 2012). Averaged across traits, the proportion of $\mathrm{ROH}$ genotypes that were below 1, 2, and $3 \mathrm{Mb}$ for LR (LW) was 2.0 (1.7), 8.3 (8.4), and $16.0 \%$ (16.2), respectively. The algorithm trapped short ROH genotypes (albeit at a low frequency). Further improvements of the algorithm in the future should focus on reducing the frequency of trapping short $\mathrm{ROH}$. Lastly, the optimal window size, rate at which the window size is reduced, and empirical $t$-statistic cutoff is likely to be dependent on the genotype density and population. The current study investigated the effectiveness of identifying unfavorable $\mathrm{ROH}$ genotypes in the context of medium-density genotype information because it is the density traditionally used in genetic evaluations. The optimal window size across different marker panel densities or sequence information should be refined based on the population studied and genotype density used.

Previous studies have investigated ROH effects by accounting for the additive genotypic value of the region in the model by either including SNP contained within the region investigated (Pryce et al., 2014) or using phenotypes that have been corrected for the additive effect (Howard et al., 2015). When using a separate model for each window, the simulation and swine data sets have illustrated that the $\mathrm{ROH}$ tagging haplotype can span many megabase pairs and is variable across animals within and across windows. Therefore, the number of SNP to include before and after the haplotype in the model to account for the additive effect for a given region is difficult to determine. More importantly, the independence between additive and dominance effects in the classical treatment (Falconer and Mackay,
1996) is, to an extent, a convenient artifact that allows orthogonality of the additive and dominance estimates. In reality, and as outlined in Huang and Mackay (2016), depending on the parameterization of the model, the variance explained by either additive, dominance or epistasis can be rearranged and placed more heavily into any of the 3 categories. This is chiefly due to the fact that 3 effects are, in a real situation, nonorthogonal to each other, so that the variance from a particular effect can be "consumed" by another effect. This is an important point because additivity and dominance are 2 intrinsically inseparable terms and if an allele is dominant over another $(a \neq 0, d \pm \mathrm{a})$, there must be additive homozygous effects ( $a \neq 0$; Huang and Mackay, 2016). The nonorthogonal relationship between additive and dominance effects has been confirmed with real data (Wellmann and Bennewitz, 2011, 2012). The nonorthogonal relationship was also observed in the current simulation study. The correlation between IIL and TDD was essentially 0 across all scenarios, although a positive correlation $(0.14)$ was observed between IIL and the EDD. Under this premise, the ability to efficiently estimate the additive and dominance effect and their potential interactions for QTL that are at a low frequency is severely reduced. Lastly, the application of the associated haplotypes identified in mating plans when correcting for the additive effect is even more complex due to a lack of clear interpretation between the combined additive and $\mathrm{ROH}$ effect for a window. Therefore, in our analysis, priority was given to estimating the genotypic value of $\mathrm{ROH}$ segments that are susceptible to displaying reduced performance based on the combined genotypic value of the given segment. Based on this premise, we make no attempt to try to understand the number of mutations present within the $\mathrm{ROH}$, the degree of epistasis that occurs, or the inheritance pattern of QTL within the segment.

\section{Conclusions}

An algorithm that identifies unfavorable haplotypes contained within a $\mathrm{ROH}$ that results in reduced phenotypic performance was developed. Across simulated and real data sets, the unfavorable haplotype tags a much larger $\mathrm{ROH}$ region that has a high probability of being IBD and as a result of recent inbreeding due to its length. Furthermore, the accuracy of prediction for the majority of the traits was greater than 0 . On the real swine data sets, multiple haplotypes were identified that had a consistent unfavorable effect across multiple traits. The use of this algorithm and the associated haplotypes allows breeding programs to more effectively identify unfavorable regions, and mating programs can be used to minimize the frequency of $\mathrm{ROH}$ occurring in the next generation. 


\section{LITERATURE CITED}

Bosse, M., H.-J. Megens, O. Madsen, Y. Paudel, L. A. F. Frantz, L. B. Schook, R. P. M. A. Crooijmans, and M. A. M. Groenen. 2012. Regions of homozygosity in the porcine genome: Consequence of demography and the recombination landscape. PLoS Genet. 8:e1003100. doi:10.1371/journal.pgen. 1003100

Browning, S. R., and B. L. Browning. 2007. Rapid and accurate haplotype phasing and missing-data inference for whole-genome association studies by use of localized haplotype clustering. Am. J. Hum. Genet.81:1084-97. doi:10.1086/521987

Charlesworth, D., and B. Charlesworth. 1987. Inbreeding depression and its evolutionary consequences. Annu. Rev. Ecol. Syst. 18:237-268. doi:10.1146/annurev.es.18.110187.001321

Chen, G. K., P. Marjoram, and J. D. Wall. 2009. Fast and flexible simulation of DNA sequence data. Genome Res. 19:136-142. doi:10.1101/gr.083634.108

Cole, J. B. 2015. A simple strategy for managing many recessive disorders in a dairy cattle breeding program. Genet. Sel. Evol. 47:94. doi:10.1186/s12711-015-0174-9

Falconer, D. S., and T. F. C. Mackay. 1996. Introduction to quantitative genetics. 4th ed. Longman Scientific and Technical, New York.

Ferenčaković, M., J. Sölkner, and I. Curik. 2013. Estimating autozygosity from high-throughput information: Effects of SNP density and genotyping errors. Genet. Sel. Evol. 45:42. doi:10.1186/1297-9686-45-42

Garrick, D. J., J. F. Taylor, and R. L. Fernando. 2009. Deregressing estimated breeding values and weighting information for genomic regression analyses. Genet. Sel. Evol. 41:55. doi:10.1186/12979686-41-55

Gilmour, A. R., B. J. Gogel, B. R. Cullis, and R. Thompson. 2009. ASReml user guide release 3.0. NSW Department of Industry and Investment, Sydney NSW 2000 Australia.

Henderson, C. R. 1976. A simple method for computing the inverse of a numerator relationship matrix used in prediction of breeding values. Biometrics 32:69-83. doi:10.2307/2529339

Howard, J. T., M. Haile-Mariam, J. E. Pryce, and C. Maltecca. 2015. Investigation of regions impacting inbreeding depression and their association with the additive genetic effect for United States and Australia Jersey dairy cattle. BMC Genomics 16:813. doi:10.1186/s12864-015-2001-7

Howard, J. T., F. Tiezzi, Y. Huang, K. A. Gray, and C. Maltecca. 2016. Characterization and management of long runs of homozygosity in parental nucleus lines and their associated crossbred progeny. Genet. Sel. Evol. 48:91. doi:10.1186/s12711-016-0269-y

Howard, J. T., F. Tiezzi, J. E. Pryce, and C. Maltecca. 2017. GenoDiver: A combined coalescence and forward-in-time simulator for populations undergoing selection for complex traits. J. Anim. Breed. Genet. (In press.) doi:10.1111/jbg. 12277

Huang, W., and T. F. C. Mackay. 2016. The genetic architecture of quantitative traits cannot be inferred from variance component analysis. PLoS Genet. 12:e1006421. doi:10.1371/journal. pgen.1006421

Keller, M. C., P. M. Visscher, and M. E. Goddard. 2011. Quantification of inbreeding due to distant ancestors and its detection using dense single nucleotide polymorphism data. Genetics 189:237249. doi:10.1534/genetics.111.130922
Kinghorn, B. P. 2011. An algorithm for efficient constrained mate selection. Genet. Sel. Evol. 43:4. doi:10.1186/1297-9686-43-4

Knol, E. F., B. Nielsen, and P. W. Knap. 2016. Genomic selection in commercial pig breeding. Anim. Front. 6(1):15-22. doi:10.2527/ af.2016-0003

Legarra, A., C. Robert-Granie, E. Manfredi, and J.-M. Elsen. 2008. Performance of genomic selection in mice. Genetics 180:611618. doi:10.1534/genetics.108.088575

Lopes, M. S., J. W. M. Bastiaansen, L. Janss, E. F. Knol, and H. Bovenhuis. 2016. Genomic prediction of growth in pigs based on a model including additive and dominance effects. J. Anim. Breed. Genet. 133:180-186. doi:10.1111/jbg.12195

Lopes, M. S., F. F. Silva, B. Harlizius, N. Duijvesteijn, P. S. Lopes, S. E. Guimarães, and E. F. Knol. 2013. Improved estimation of inbreeding and kinship in pigs using optimized SNP panels. BMC Genet. 14:92. doi:10.1186/1471-2156-14-92

Perez, P., and G. de los Campos. 2014. Genome-wide regression and prediction with the BGLR statistical package. Genetics 198:483-495. doi:10.1534/genetics.114.164442

Pryce, J. E., M. Haile-Mariam, M. E. Goddard, and B. J. Hayes. 2014. Identification of genomic regions associated with inbreeding depression in Holstein and Jersey dairy cattle. Genet. Sel. Evol. 46:71. doi:10.1186/s12711-014-0071-7

Putz, A. M., F. Tiezzi, C. Maltecca, K. A. Gray, and M. T. Knauer. 2015. Variance component estimates for alternative litter size traits in swine. J. Anim. Sci. 93:5153-5163. doi:10.2527/ jas.2015-9416

Saura, M., A. Fernández, L. Varona, A. I. Fernández, M. Á. R. de Cara, C. Barragán, and B. Villanueva. 2015. Detecting inbreeding depression for reproductive traits in Iberian pigs using genome-wide data. Genet. Sel. Evol. 47:1. doi:10.1186/s12711014-0081-5

Simmons, M. J., and J. F. Crow. 1977. Mutations affecting fitness in Drosophila populations. Annu. Rev. Genet. 11:49-78. doi:10.1146/annurev.ge.11.120177.000405

VanRaden, P. M. 2008. Efficient methods to compute genomic predictions. J. Dairy Sci. 91:4414 4423. doi:10.3168/jds.2007-0980

VanRaden, P. M., K. M. Olson, D. J. Null, and J. L. Hutchison. 2011. Harmful recessive effects on fertility detected by absence of homozygous haplotypes. J. Dairy Sci. 94:6153-6161. doi:10.3168/ jds.2011-4624

Wellmann, R., and J. Bennewitz. 2011. The contribution of dominance to the understanding of quantitative genetic variation. Genet. Res. 93:139-154. doi:10.1017/S0016672310000649

Wellmann, R., and J. Bennewitz. 2012. Bayesian models with dominance effects for genomic evaluation of quantitative traits. Genet. Res. 94:21-37. doi:10.1017/S0016672312000018

Wolc, A., C. Stricker, J. Arango, P. Settar, J. E. Fulton, N. P. O’Sullivan, R. Preisinger, D. Habier, R. Fernando, D. J. Garrick, S. J. Lamont, and J. C. Dekkers. 2011. Breeding value prediction for production traits in layer chickens using pedigree or genomic relationships in a reduced animal model. Genet. Sel. Evol. 43:5. doi:10.1186/1297-9686-43-5

Xiang, T., O. F. Christensen, Z. G. Vitezica, and A. Legarra. 2016. Genomic evaluation by including dominance effects and inbreeding depression for purebred and crossbred performance with an application in pigs. Genet. Sel. Evol. 48:92. doi:10.1186/ s12711-016-0271-4 
Reproduced with permission of copyright owner. Further reproduction prohibited without permission. 